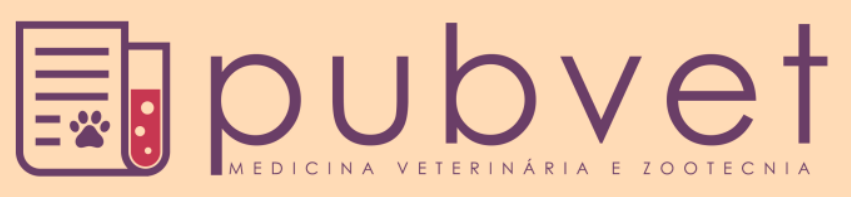

HTTP://DX.DOI.ORG/10.22256/PUBVET.V11N9.917-922

\title{
Torção primária do baço em cães: Relato de caso
}

\author{
Mariela Sousa Gomes ${ }^{1 *}$, João Macedo de Sousa², Sabrina Barros Araújo ${ }^{3}$, Francisco \\ Lima Silva ${ }^{2}$, Rebecca Tavares Lima ${ }^{1}$, Raquel Albuquerque Silva ${ }^{1}$, Gerson Tavares \\ Pessoa $^{1}$, Marinna Nérica do Nascimento e Silva ${ }^{1}$
}

${ }^{l}$ Médico(a) Veterinária. Residente do Hospital Veterinário Universitário da Universidade Federal do Piauí. Teresina - PI, Brasil. ${ }^{2}$ Médico Veterinário. Professor da Universidade Federal do Piauí, Departamento de Clínica e Cirurgia Veterinária. Teresina - PI, Brasil.E-mail: smacedo@ufpi.edu.br

${ }^{3}$ Médica Veterinária do Hospital Veterinário Universitário (HVU) da Universidade Federal do Piauí (UFPI). Teresina - PI, Brasil.

*Autor para correspondência, E-mail: mariela.gomes@hotmail.com

\begin{abstract}
RESUMO. Torção esplênica é uma condição rara em cães. A rotação ocorre em seu pedículo vascular e geralmente ocorre concomitantemente à dilatação vólvulo gástrica, porém a torção primária esplênica é rara. Segundo a literatura essa enfermidade acomete normalmente cães de grande porte e raças de peito profundo. Os sinais clínicos, achados laboratoriais e radiográficos são inespecíficos e inconclusivos para o diagnóstico preciso de torção esplênica. Assim, a ultrassonografia juntamente com o uso do Doppler colorido tem sido relatada como ferramentas fundamentais para a sugestão do diagnóstico de torção esplênica, que é confirmado através de laparotomia exploratória, geralmente seguida de esplenectomia total como forma de tratamento. Este estudo relata o caso de um cão da raça Pit Bull, três anos de idade, cujo exame ultrassonográfico foi uma ferramenta de grande valor diagnóstico para detecção desta enfermidade.
\end{abstract}

Palavras chave: Baço, torção esplênica, ultrassonografia esplênica

\section{Primary splenic torsion in dogs: Case report}

ABSTRACT. Splenic torsion is a rare condition in dogs. The torsion occurs in the vascular pedicle and usually occurs concomitantly with dilatation gastric volvulus, but the primary splenic torsion is rare. According to the literature, this disease usually affects dogs of big size and breeds of deep chest. Clinical signs, laboratory and radiographic findings are nonspecific and inconclusive for the accurate diagnosis of splenic torsion. Thus, ultrasound along with the use of color Doppler has been reported as fundamental tools for the suggestion of the diagnosis of splenic torsion, which is confirmed by exploratory laparotomy, usually followed by total splenectomy as a form of treatment. This study reports the case of a 3-year-old Pit Bull dog whose ultrasound examination was a tool of great diagnostic value for the detection of this disease.

Keywords: Spleen, splenic torsion, splenic ultrasonography

\section{Torsión primaria del bazo en los perros: Reporte de un caso}

RESUMEN. Torsión esplénica es una enfermedad poco frecuente en los perros. La rotación se produce en su pedículo vascular y por lo general se produce simultáneamente con la dilatación vólvulo gástrico, pero la torsión primaria esplénica es rara. Según la literatura esta enfermedad normalmente afecta a los perros de tamaño grande y razas de pecho profundo. Los signos clínicos, de laboratorio y los hallazgos radiológicos son inespecíficos y no concluyentes para el diagnóstico preciso de la torsión esplénica. Por lo tanto, la ultrasonografía junto con el uso del Doppler a color ha sido reportada como 
herramientas fundamentales para la indicación del diagnóstico de torsión esplénica, que se confirma por laparotomía exploratoria, generalmente seguido por la esplenectomía total como tratamiento. Este estudio reporta el caso de un perro de raza Pit Bull, tres años de edad, cuyo examen ultrasonográfico fue una valiosa herramienta de diagnóstico para la detección de esta enfermedad.

Palabras clave: bazo, torsión esplénica, ultrasonido esplénico

\section{Introdução}

O baço está situado no quadrante abdominal cranial esquerdo, porém sua localização exata pode depender da posição e tamanho de outros órgãos abdominais (Fossum, 2014). Paralelo à curvatura maior do estômago, sua cabeça é fixa ao estômago pelo ligamento gastroesplênico (Thrall, 2013). O baço pode ser acometido por doenças inflamatórias, circulatórias, hematológicas e neoplásicas, tanto como sítio primário, quanto metastático (Slatter, 2007). Dentre as suas enfermidades possíveis, existe a torção. Esta ocorre quando o baço gira ao redor do seu eixo mesentérico, resultando em completa oclusão da drenagem venosa e eventual oclusão arterial (Thrall, 2013). Geralmente vem em associação com volvo gástrico, mas pode vir de forma espontânea, sendo mais rara. A causa da torção esplênica primária não está clara e pode estar relacionada a anormalidades congênitas ou rompimento do ligamento gastroesplênico ou esplenocólico (Konde et al., 1989, Fossum, 2014). Segundo Azevedo et al. (2011), cães de grande porte ou com abdome profundo e mais espaçoso, apresentam com maior frequência a doença devido terem uma maior movimentação dos órgãos abdominais (Azevedo et al., 2011).

As manifestações clínicas são inespecíficas, podendo ocorrer sinais de dor abdominal, aumento do pulso e da frequência respiratória, palidez das mucosas, vômito, anorexia, perda de peso, distenção abdominal, depressão e letargia (Konde et al., 1989, Neath et al., 1997, Azevedo et al., 2011, Ortiz et al., 2016). Exames complementares como radiografia e ultrassonografia devem ser utilizados; porém a laparotomia exploratória é a única forma de diagnóstico definitivo para a torção esplênica isolada (Konde et al., 1989, Goldsmid et al., 1994).

A torção esplênica requer tratamento de emergência sendo este a esplenectomia (Konde et al., 1989, Rial et al., 2010, Azevedo et al., 2011, Fossum, 2014, Ortiz et al., 2016) acompanhado de conduta para necessária estabilização do animal para o procedimento cirúrgico (Fossum, 2014).

\section{Material e Métodos}

Uma cadela da raça Pit Bull, de três anos, sem relato de doença anterior, massa corporal de 23,6 $\mathrm{kg}$, foi encaminhada ao Hospital Veterinário Universitário da Universidade Federal do Piauí (HVU-UFPI) para atendimento. Apresentava sangramento vaginal, apatia, hiporexia há três dias, aumento de volume abdominal e presença de ectoparasitas. O proprietário do animal referiu que a cadela apresentava normoquesia, normodipsia e ausência de vômitos. Relatou ainda que administrava anticoncepcional ao animal. Durante a anamnese constatou-se que $o$ animal alimentavase apenas uma vez ao dia. Ao exame clínico o grande aumento de volume abdominal chamou a atenção. Devido ao proprietário relatar uso rotineiro de anticoncepcional no animal e esta ter cruzado recentemente e estar apresentando leve sangramento vaginal, foi solicitado exame ultrassonográfico suspeitando-se de alguma alteração em nível de útero.

Além do exame ultrassonográfico foram solicitados exames sanguíneos, hemograma e avaliação bioquímica sérica: ureia, creatinina, ALT/TGP, fosfatase alcalina, proteína total, albumina e globulina. O hemograma completo revelou anemia normocítica hipocrômica (hematócrito $18,9 \%$ ) e plaquetas $\left(173 \times 10^{3} \mu \mathrm{L}\right)$ abaixo do valor mínimo para cães. No leucograma constatou-se leucocitose $(76.100 \mathrm{cel} / \mu \mathrm{L})$, Neutrofilia (Bastonetes 761/ $\mu \mathrm{L}$ e Segmentados $60.119 / \mu \mathrm{L}$ ) com desvio à esquerda regenerativo, Linfopenia, Eosinopenia e Monocitose. Não foram observados hematozoários. Na avaliação bioquímica sérica, os valores de ureia $(362$ $\mathrm{mg} / \mathrm{dL})$, creatinina $(6,7 \mathrm{mg} / \mathrm{dL})$, ALT $(80 \mathrm{U} / \mathrm{L}) \mathrm{e}$ FA (199 U/L) estavam aumentados.

O paciente foi encaminhado para o setor de diagnóstico por imagem do HVU - UFPI e submetido à varredura abdominal, após a devida tricotomização e colocação de gel condutor. Usouse o transdutor micro-convexo CN4-9 de frequência 4-9 MHz, em aparelho de Ultrassom Medison SonoAce R3. No exame foi verificado severo aumento do baço (esplenomegalia) ocupando não só o lado esquerdo, como direito do 
abdômen, impedindo a devida visualização de outros órgãos como o fígado. A ecogenicidade do parênquima esplênico apresentava-se diminuída difusa (hipoecoico), a textura difusamente com aparência rendilhada (Figura 1).

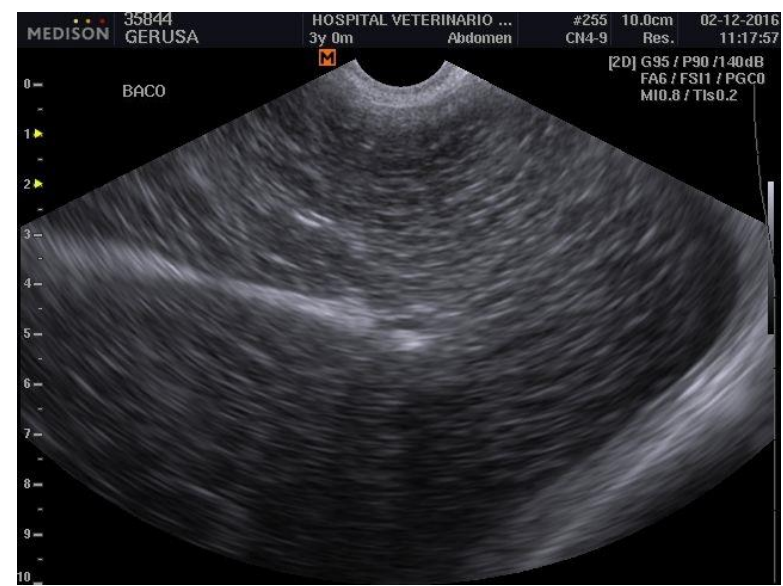

Figura 1. Imagem ultrassonográfica evidenciando severa esplenomegalia e parênquima esplênico com ecogenicidade diminuída (hipoecoico) e textura rendilhada difusa. Fonte: Universidade Federal do Piauí, Hospital Veterinário Universitário, Setor de Diagnóstico por Imagem, 2016.

Observou-se um aumento de ecogenicidade na gordura circundante formando uma imagem triangular hiperecoica perivenosa, localizada no hilo (Figura 2). No exame modo B com Doppler colorido não se verificou presença de fluxos sanguíneos nas veias esplênicas (Figura 2).

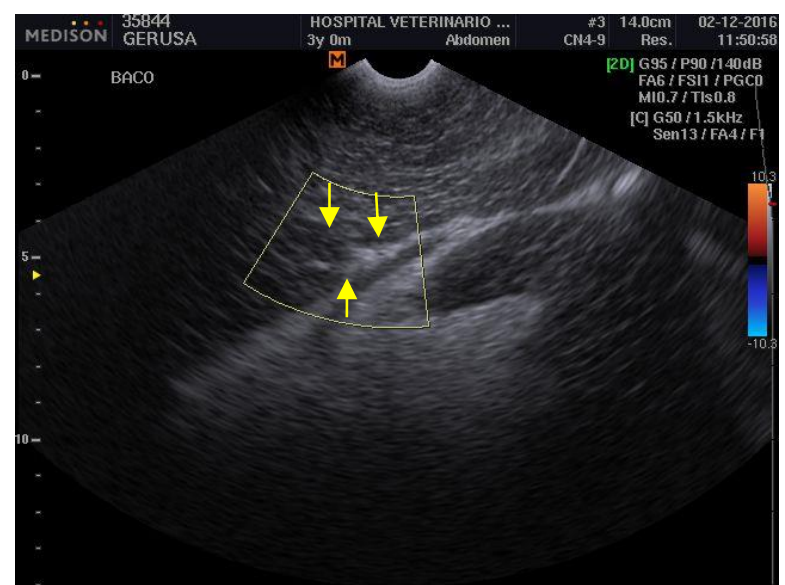

Figura 2. Imagem ultrassonográfica em modo Doppler demonstrando ausência de fluxo sanguíneo no baço e evidenciando um aumento de ecogenicidade na gordura circundante formando uma imagem triangular hiperecoica perivenosa, localizada no hilo (setas amarelas). Fonte: Universidade Federal do Piauí, Hospital Veterinário Universitário, Setor de Diagnóstico por Imagem, 2016.

Havia grande presença de efusão peritoneal (Figura 3). Os sinais descritos foram sugestivos para torção e infarto esplênico. $\mathrm{O}$ útero não foi visualizado ao exame devido à grande extensão do baço dentro da cavidade abdominal. Realizou-se radiografia abdominal simples, porém devido à grande quantidade de efusão peritoneal, os órgãos abdominais não puderam ser evidenciados (Figura 4).

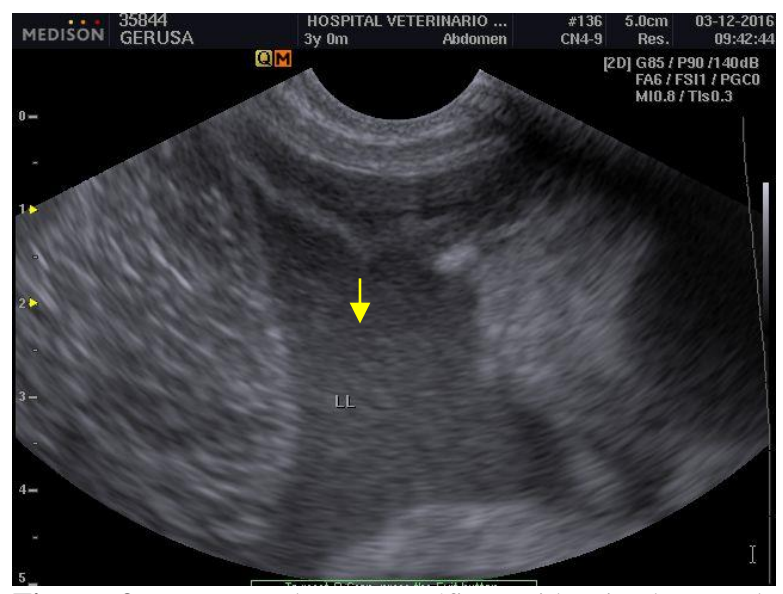

Figura 3. Imagem ultrassonográfica evidenciando grande presença de líquido livre na cavidade abdominal (seta amarela). Fonte: Universidade Federal do Piauí, Hospital Veterinário Universitário, Setor de Diagnóstico por Imagem, 2016.

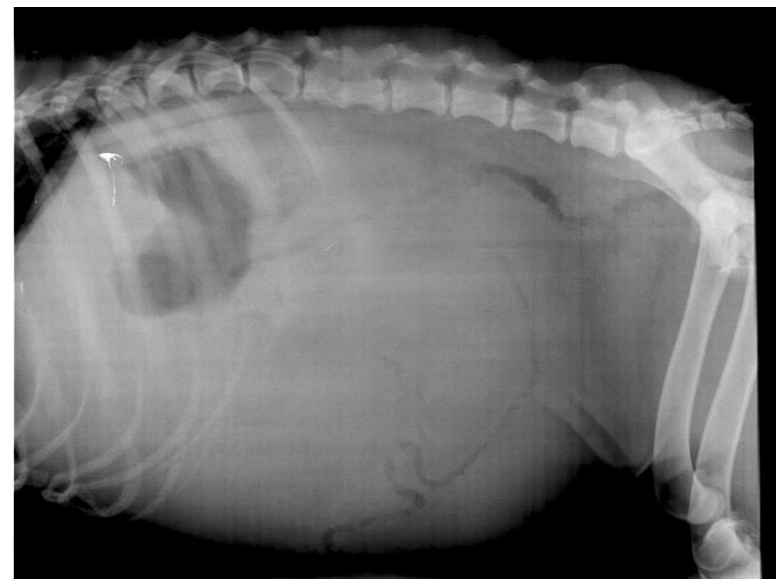

Figura 4. Imagem radiográfica abdominal simples na posição lateral esquerda-direita demonstrando grande aumento de radiopacidade água difusa abdominal, dificultando a visualização dos órgãos (efusão peritoneal). Fonte: Universidade Federal do Piauí, Hospital Veterinário Universitário, Setor de Diagnóstico por Imagem, 2016.

O paciente foi encaminhado para laparotomia exploratória. $\mathrm{Na}$ laparotomia exploratória, confirmou-se o quadro de torção esplênica. Foi realizada a retirada do baço, e este se apresentava escurecido, com aspecto desvitalizado, retorcido, envolto em porção do omento (Figura 5). Não foi observada alteração de vitalidade do estômago. Também não foram visualizadas alterações em útero. $\mathrm{O}$ baço foi encaminhado para exame histopatológico, que identificou intensa necrose esplênica associada a intensa hemorragia difusa. 


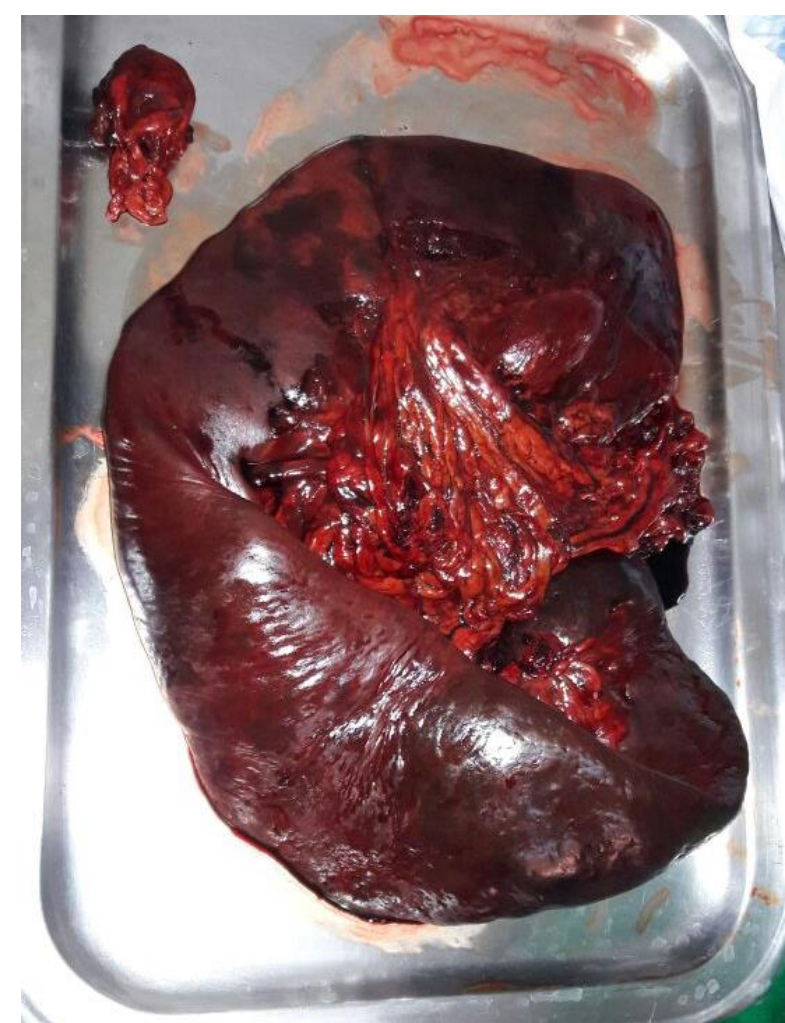

Figura 5. Baço retorcido sobre si mesmo, envolto por omento desvitalizado, após ser removido da cavidade abdominal. Fonte: Universidade Federal do Piauí, Hospital Veterinário Universitário, 2016.

\section{Resultados e Discussão}

Alguns casos de torção esplênica primária já foram relatados na literatura, porém sua ocorrência continua sendo considerada como uma enfermidade incomum. Acomete principalmente cães de porte grande e gigante, com abdome profundo e mais espaçoso (Fossum, 2014). A maioria dos relatos descritos refere-se a casos acontecidos com cães das raças Pastor Alemão e Dogue Alemão (Maxie et al., 1970, Stead et al., 1983, Konde et al., 1989, Neath et al., 1997, Simeonova et al., 2007, Azevedo et al., 2011). No presente trabalho relata-se um caso ocorrido com um cão da raça Pit Bull; sendo esta raça como afetada pela mesma enfermidade por Azevedo et al. (2011). Azevedo et al. (2011) ressaltaram que a raça Pit Bull vem se tornando popular nos últimos anos, estando aumentando seu número, justificando o aumento da incidência desta enfermidade nesta raça. Outro ponto sugerido como agravante para a raça Pit Bull estar susceptível a torção esplênica seja em relação ao seu comportamento, pois pulam grandes alturas constantemente (Azevedo et al., 2011).

Existem diferentes hipóteses sobre a etiologia desta enfermidade. Está mais comumente associada à dilatação vólvulo-gástrica do que a doenças esplênicas, mas apesar de ser rara, pode ocorrer sem histórico de doença gástrica e esplênica prévia (Mai, 2006). Um manejo alimentar feito pelo proprietário, onde os animais alimentam-se apenas uma vez ao dia, como relatado no presente trabalho, e provavelmente com quantidades acima da recomendada pode sugerir envolvimento da dilatação gástrica (Azevedo et al., 2011). Em casos que tenha ocorrido uma torção esplênica sem evidências de dilatação ou torção gástrica é sugerido por Maxie et al. (1970) que alterações gástricas tenham ocorrido e tenham sofrido resolução espontânea. Em um estudo, Neath et al. (1997) identificaram que $21 \%$ dos cães acometidos com torção esplênica tiveram episódios de dilatação vólvulogástrica meses antes, corroborando com a teoria de Maxie et al. (1970).

Goldsmid et al. (1994) sugeriram que repetidos episódios de dilatação gástrica e consequente torção parcial deste órgão causam o estiramento e o afrouxamento dos ligamentos gastroesplênicos, ocasionando mobilidade e rotação esplênica. E entre os principais sinais clínicos verifica-se a síndrome do abdome agudo, que é caracterizada por um início repentino de sinais, tais como distensão abdominal, dor, anorexia e vômito (Fossum, 2014). Além desses sinais, outros podem ser observados, como a esplenomegalia que é resultante de um fluxo arterial contínuo seguido de uma obstrução venosa que determina o aumento do órgão (Maxie et al., 1970). As manifestações clínicas encontradas na cadela aqui relatadas corroboram com os achados de outros autores (Maxie et al., 1970, Konde et al., 1989, Neath et al., 1997, Mai, 2006, Rial et al., 2010, Azevedo et al., 2011, Ortiz et al., 2016).

O paciente apresentava anemia, assim como relatado por outros autores (Maxie et al., 1970, Konde et al., 1989, Neath et al., 1997, Mai, 2006, Rial et al., 2010, Azevedo et al., 2011, Ortiz et al., 2016). A anemia é resultante do sequestro de hemácias pelo baço torcido e da inflamação em curso, desde que descartadas hemoparasitose e anemia hemolítica imunomediada, segundo Schnier (2010). O leucograma apresentou leucocitose corroborando com outros autores (Maxie et al., 1970, Konde et al., 1989, Neath et al., 1997, Mai, 2006, Rial et al., 2010, Azevedo et al., 2011, Ortiz et al., 2016). Neste caso, relacionase a leucocitose com a inflamação associada à baixa perfusão do parênquima esplênico e peritonite localizada secundária à torção esplênica, que foi demonstrada pelo mesentério 
reativo e pela efusão peritoneal, visualizados na ultrassonografia (Ortiz et al., 2016).

Ao exame ultrassonográfico verificou-se um grande aumento de volume esplênico, estendendose por grande parte do abdome. Com parênquima difusamente hipoecoico e com aparência rendilhada, aumento de ecogenicidade na gordura e mesentério circundantes, associados à efusão peritoneal sendo achados compatíveis aos descritos por Penninck and D'Anjou (2011) para casos de torção esplênica. A ultrassonografia com Doppler colorido possibilitou a detecção de ausência de fluxo sanguíneo no parênquima esplênico. Mai (2006) enfatiza que o uso do Doppler facilita o diagnóstico, demonstrando a falta de vascularização; porém, a ausência de fluxo sanguíneo também pode ocorrer devido à trombose da veia esplênica.

Em relação ao exame radiográfico, no presente trabalho apenas evidenciou-se grande aumento de radiopacidade água de forma difusa em região abdominal, sugestivo para efusão peritoneal. A grande presença de líquido intra-abdominal comprometeu a qualidade do exame radiográfico como observado por Konde et al. (1989). Assim como relatado por Rial et al. (2010), Azevedo et al. (2011), Ortiz et al. (2016), para diagnóstico diferencial realizou-se a laparotomia exploratória, verificando-se a torção esplênica. Quanto ao tratamento, verificou-se que o tratamento de eleição é a esplenectomia total. Após esplenectomia, o órgão foi encaminhado para o setor de patologia do HVU-UFPI, que identificou intensa necrose esplênica associada à intensa hemorragia difusa, corroborando com $\underline{\text { Saunders et }}$ al. (1998), Mai (2006), Ortiz et al. (2016).

\section{Conclusão}

A raça Pit Bull parece ser predisposta a ocorrência de torção esplênica devido seu comportamento e o seu grande porte. A baixa frequência alimentar também é verificada como fator influenciador, uma vez que pode causar dilatação ou torção gástrica, enfermidades as quais predispõem a ocorrência de uma torção esplênica. O diagnóstico é baseado em sinais clínicos associados a exames complementares. Achados laboratoriais e exames radiográficos foram inconclusivos no diagnóstico de torção esplênica. Dessa forma, também se deve associar a avaliação clínica com exame de ultrassonografia abdominal com avaliação de fluxo sanguíneo pelo Doppler ou Power Doppler colorido. Como diagnóstico diferencial, a laparotomia exploratória é fundamental, e como tratamento a esplenectomia total deve ser realizada.

\section{Referências Bibliográficas}

Azevedo, F. D., Veiga, C. C. P., Scott, F. B., Fernandes, J. I., Ramos, A. S. \& Mendonça, E. C. L. 2011. Torção primária do baço em cães Relato de caso. Revista Brasileira de Medicina Veterinária, 33, 89-94.

Fossum, T. W. 2014. Cirurgia de pequenos animais, 4 edn. Elsevier Brasil, São Paulo.

Goldsmid, S. E., Davis, P. \& Pechman, R. 1994. Successful derotation of a splenic torsion in a racing greyhound. Journal of Small Animal Practice, 35, 112-115.

Konde, L. J., Wrigley, R. H., Lebel, J. L., Park, R. D., Pugh, C. \& Finn, S. 1989. Sonographic and radiographic changes associated with splenic torsion in the dog. Veterinary Radiology \& Ultrasound, 30, 41-45.

Mai, W. 2006. The hilar perivenous hyperechoic triangle as a sign of acute splenic torsion in dogs. Veterinary Radiology \& Ultrasound, 47, 487-491.

Maxie, M. G., Reed, J. H., Pennock, P. W. \& Hoff, B. 1970. Case report. Splenic torsion in three great danes. The Canadian Veterinary Journal, 11, 249-255.

Neath, P. J., Brockman, D. J. \& Saunders, H. M. 1997. Retrospective analysis of 19 cases of isolated torsion of the splenic pedicle in dogs. Journal of Small Animal Practice, 38, 387-392.

Ortiz, B. C., Oliveira, C. M., Teixeira, L. G., Koch, M. C. \& Muller, V. S. 2016. Torção esplênica primária em um cão: relato de caso. Arquivo Brasileiro de Medicina Veterinária e Zootecnia, 68, 1195-1200.

Penninck, D. G. \& D'Anjou, M. A. 2011. Atlas de ultrassonografia de Pequenos animais. Guanabara Koogan, Rio de Janeiro.

Rial, A. F., Walesca, S., Yamanaka, V. S., Cassanego, L. H., Wilmsen, M. O., Oliveira, S., Meirelles, C. F., Martins, L. G. A. \& Lima, M. C. 2010. Relato de caso: Torção de coto esplênico PUBVET, 4, Ed. 138.

Saunders, H. M., Neath, P. J. \& Brockman, D. J. 1998. B-mode and Doppler utrasound imaging of the spleen with canine splenic torsion: a retrospective evaluation. Veterinary Radiology \& Ultrasound, 39, 349-353. 
Schnier, L. M. 2010. A case of splenic torsion with progressive anemia and thrombocytopenia. The Canadian Veterinary Journal, 51, 527529.

Simeonova, G., Simeonov, R. \& Roussenov, A. 2007. Uncommon cause of acute abdômen in a dog: torsion of the spleen-case report and review. Trakia Journal of Sciences, 5, 64-68.

Slatter, D. H. 2007. Manual de cirurgia de pequenos animais. Manole, São Paulo.

Stead, A. C., Frankland, A. L. \& Borthwick, R. 1983. Splenic torsion in dogs. Journal of Small Animal Practice, 24, 549-554.
Thrall, D. E. 2013. Textbook of veterinary diagnostic radiology. Elsevier Health Sciences, Philadelphia.

\section{Article History:}

Received 26 April 2017

Accepted 2 June 2017

Available online 8 August 2017

License information: This is an open-access article distributed under the terms of the Creative Commons Attribution License 4.0, which permits unrestricted use, distribution, and reproduction in any medium, provided the original work is properly cited. 